\title{
An investigation into the effects of different types of exercise on the maintenance of approach motivation levels
}

Lowenstein, JAS

http://hdl.handle.net/10026.1/10595

10.1016/j.mhpa.2015.08.002

Mental Health and Physical Activity

Elsevier BV

All content in PEARL is protected by copyright law. Author manuscripts are made available in accordance with publisher policies. Please cite only the published version using the details provided on the item record or document. In the absence of an open licence (e.g. Creative Commons), permissions for further reuse of content should be sought from the publisher or author. 
An Investigation into the Effects of Different Types of Exercise on the Maintenance of Approach Motivation Levels

J.A.S. Lowenstein, K.Wright, A. Taylor ${ }^{1}$, \& N. J. Moberly

University of Exeter, Washington Singer Building, Perry Road, Exeter, United Kingdom.

EX4 4QG.

Corresponding author. Email joseph.lowenstein@nhs.net +447793556976 ,

30 Paisley Road, Southbourne, Bournemouth, Dorset. United Kingdom. BH65ET

Highlights:

- Research into the benefits of exercise for individuals with experiencing hypomania is limited

- We looked to investigate the impact of different intensities of exercise on the maintenance of AM levels

- Vigorous exercise significantly increased individuals' AM levels in comparison to moderate or no exercise.

- The results have implications for the possible risks associated with engaging in different intensities of exercise during a hypomanic episode.

\footnotetext{
${ }^{1}$ Present address: Plymouth University Peninsula Schools of Medicine \& Dentistry, Room N32, ITTC Building, Tamar Science Park, Derriford, Plymouth, Devon, United Kingdom. PL6 8BX
} 


\section{Abstract}

\section{Background}

This study looked to investigate the interaction between exercise and approach motivation (AM) levels in a non-clinical sample as a first step towards investigating the impact of acute exercise upon hypomanic states within Bipolar Disorder. The Behavioural Activation System (BAS) dysregulation theory proposes that AM levels in individuals with Bipolar Disorder, are hyper-reactive to relevant cues and prone to fluctuation such that excessive levels underpin hypomania / mania. We hypothesise that exercise may interact with high AM levels to further increase AM levels in both the general population and individuals with $\mathrm{BD}$, with this effect being exacerbated in the latter group. As an initial test of this theory we explore the impact of moderate and vigorous exercise and sedentary activity upon AM in an unselected student sample. We also tested the extent to which hypomania vulnerability predicts the impact of exercise. Method

Participants were recruited from a University student population. After completing a measure of hypomanic personality traits, 61 participants completed a task designed to induce higher levels of AM before taking part in one of three 15 minute activities (sedentary, moderate exercise or vigorous exercise). AM levels as well as variables relevant to hypomanic symptoms were measured prior to and post AM induction, at 5 minute intervals during the activities and twice during a recovery period.

Results

Vigorous exercise significantly increased individuals' AM levels in comparison to moderate or no exercise. No association was found between hypomania vulnerability and exercise impact.

\section{Conclusions}

These results provide a first step in investigating the possible risks associated with engaging in different intensities of exercise during a hypomanic episode. Any recommendations within this study should however be taken in light of the limitations identified. Further research replicating these results with a larger sample and among individuals with Bipolar Disorder is recommended.

Keywords: Bipolar Disorder, behavioural activation system, behavioural approach system, approach motivation, exercise. 
An Investigation into the Effects of Different Types of Exercise on the Maintenance of Approach Motivation Levels

Despite the evidence that exercise can have a positive effect on uni-polar depression (Cooney et al, 2013), limited research exists on the effects of exercise for individuals with Bipolar Disorder (BD). This was highlighted in a systematic review that incorporated a number of electronic databases from inception to January 2009. Of the 484 articles retrieved, only six studies were identified that looked quantitatively at the effects of exercise upon the physical or mental health of individuals with Bipolar Disorder, and of these, none were adequately-powered RCTs (Wright, Everson-Hock, \& Taylor, 2009). Since then, Sylvia et al (2013) have reported a negative correlation between increased levels of exercise and depressive mood, with higher levels of exercise associated with increased manic symptoms and a brief review by Stanton et al (2014) concluded that low to moderate intensity exercise for 30 to 40 minutes, three to four times a week, over at least 9 to 12 weeks, is likely to be beneficial for people with affective disorders in general.

The current guidelines by the National Institute of Clinical Excellence (NICE) state that effective simple treatments for depression can play a useful role in treating BD because depressive symptoms are present in around one third of these individuals (Judd et al., 2002). Therefore, exercise is recommended on this basis and in relation to weight gain associated with the side effects of medication (NICE, 2006).

Very little is known however, about the interaction between exercise and acute hypomania or mania: NICE states that there is "potential for exercise to be both helpful and harmful in mania but there is no research evidence to support either scenario" ( $p .390$, NICE, 2006). Wright, and colleagues (2011) reported findings from semi structured interviews with 25 individuals with a diagnosis of $\mathrm{BD}$. 
Pertinent to the question of risks versus benefits of exercise in this population, one major theme, 'exercise as a double-edged sword' found exercise effects to depend upon variables such as mood state at time of the exercise or the intensity of exercise engaged in. The possibility of an 'upward spiral' was reported in which the onset of manic symptoms leads to an increase in the amount or intensity of the exercise which in turn exacerbates symptoms further.

One possible mechanism by which exercise may exacerbate hypomanic symptoms is through its effects upon the Behavioural Activation System (BAS). The BAS is proposed to be an internal system that governs approach motivation (AM; Gray, 1987). AM can be defined as the energization of behaviour by, or the direction of behaviour toward, positive stimuli (objects, events, possibilities; Elliot, 1999). It is the process involved when a person takes action towards anything that gives them happiness, pleasure, or joy. Its basic adaptive function is to ensure that organisms obtain resources (e.g. food, shelter, companionship) and is therefore essential to survival of the individual and the species (Watson, Wiese, Vaidya, \& Tellegen, 1999). The BAS dysregulation theory (Depue \& Iacono, 1989; Depue, Krauss, \& Spoont, 1987) proposes that individuals with BD have a BAS that is overly sensitive to goal orientated cues. This over-sensitivity results in large fluctuations in the activation and deactivation of the BAS which is reflected in the symptoms of BD. For example, when vulnerable individuals experience events involving rewards or goal striving the overly sensitive BAS becomes excessively activated over an extended period of time, resulting in manic symptoms, such as excessive goal-directed behaviour, increased energy, optimism, and euphoria and a reduction in the need for sleep (Depue, \& Iacono, 1989; Urosevic, Abramson, Harmon-Jones, \& Alloy, 2008). A number of studies have tested predictions of the theory amongst individuals with Bipolar Disorder and vulnerable populations, and overall their findings support the hypothesis that Bipolar Disorder is associated with, and contributed to, 
by dysregulation of the approach system (for reviews see Alloy \& Abramson, 2010; Urosevic et al., 2008).

We propose that exercise is both a potential trigger of the BAS, and a likely output of high BAS activity. During an initial period of heightened AM, exercise may be preferentially selected by some individuals because it is rewarding (Reed \& Ones, 2006); this because it often involves pursuit and attainment of exercise-related goals, or due to more direct effects of exercise upon affective valence. Consequently, the BAS is stimulated further. As such exercise, or certain forms of exercise, may contribute to an upward spiral of approach motivation and activity. Whilst this positive feedback loop may occur to some extent in individuals without Bipolar Disorder, we propose that it is particularly pernicious in those with Bipolar Disorder, as it has been hypothesised that BAS regulatory strength is reduced in this group.

From the proposed model, and the findings of Wright and colleagues (2011) it would be expected that moderate and vigorous exercise will lead to greater stimulation of the BAS and hence increases in AM levels in comparison to no exercise. However, previous literature within the remit of exercise and affect has shown that vigorous exercise leads initially to decreases in positive affect in comparison to moderate exercise (see review by Ekkekakis, Parfitt, \& Petruzzello, 2011). This may suggest that individuals may demonstrate reduced AM levels immediately following vigorous exercise because of the close relationship between AM and positive affect. This is an important area of further investigation as it may have implications for the types of exercise that are least likely to promote symptom exacerbation in hypomania.

To date, no studies have investigated the impact of exercise intensity upon hypomanic mood. Prior to conducting research of this nature, which is associated with significant practical and ethical implications, we sought to establish whether exercise exacerbates high 
levels of approach motivation in the general population, relative to sedentary activity. To our knowledge, research upon the impact of exercise on emotional or motivational state in the general population has tended to focus upon consequences for mood and arousal level, rather than approach motivation specifically (e.g. Ekkekakis \& Petruzzello, 1999; Hall, Ekkekakis \& Petruzzello, 2002). Therefore there is a paucity of information concerning the interaction between approach motivation and exercise even within non-clinical samples. As described above, we predict that exercise will exacerbate high AM in the general population, and that it will have a more pronounced effect in those vulnerable to hypomanic states.

The aim of the current study was therefore to investigate the effect of different intensities of exercise on the maintenance of AM levels amongst individuals already in a state of heightened AM. The primary hypothesis (1) was that following an initial increase in AM, relative to individuals undergoing subsequent sedentary activity, those taking part in subsequent exercise will show increased approach motivation. To investigate the impact of exercise upon manic-like symptoms we extended this prediction to secondary variables chosen to reflect key features of mania (American Psychiatric Association, 2013); b) high activation positive affect, c) perceived thought speed, d) feelings associated with mania, and e) motor activity following exercise. Additionally, we investigated the interaction between hypomanic personality traits and the effects of exercise upon AM. We hypothesised that (2) the relationships predicted in hypothesis one will be moderated by the presence of hypomanic traits such that they will be stronger in individuals reporting higher levels of hypomanic traits. We also predicted that following an initial increase in approach motivation, (3) there will be a positive relationship between hypomanic traits and time taken for approach motivation levels to return to baseline and (4) there will be a positive relationship between hypomanic traits and participants' reported desire to continue with exercise. 


\section{Method}

\section{Participants}

Participants were recruited from the student population of a University in the South West of England. Participants were required to be aged 18 or over and able to read and understand English. For ethical reasons, participants were excluded if they had physical health problems that would make exercise dangerous, or attained a score of seven or above on the depression subscale of the Hospital Anxiety and Depression Scale (HADS: Zigmond, \& Snaith, 1983), or six or above on the Altman Self-Rating Mania Scale (ASRM: Altman, Hedeker, Peterson, Davis, 1997) in line with established cut-off scores for potentially clinically significant symptom levels.. They were also excluded if they did not respond to the laboratory AM induction procedure by demonstrating an increase in AM levels of at least one point as measured by the Behavioural Engagement Scale (BES) detailed below.

\section{Materials}

\section{Baseline measures.}

Hospital Anxiety and Depression Scale (HADS; Zigmond, \& Snaith, 1983). A 14 item self-rating measure for anxiety/depression over the last week. Only the depression subscale (HADS-D) was used with potential scores ranging from 0 to 21 . Good internal consistency has been found for the HADS-D $(\alpha=.82)$ and good correlation with other commonly used measures has been reported (r=.49-.83; Bjelland, Dahl, Haug, \& Neckelmann, 2002).

Altman Self-Rating Mania Scale (ASRM; Altman, Hedeker, Peterson, Davis, 1997). A five item measure for rating symptoms of mania over the last week on a scale from 0-4 based on increasing severity. It includes the following symptoms; elevated/euphoric mood, increased self-esteem, decreased need for sleep, pressured speech and psychomotor agitation. 
EXERCISE AND APPROACH MOTIVATION

The test-retest reliability of the ASRM is significant ( $r=.86)$ and it is reported to be significantly correlated with other mania measures ( $r=.718-$.766; Altman et al., 1997).

Hypomanic Personality Scale (HPS, Eckblad, \& Chapman, 1986). A 48 item true/false self-report questionnaire used to assess stable hypomanic traits. High scores on the HPS have been shown to predict symptoms of BD in the short term (Eckblad, \& Chapman, 1986) and over the long-term (Blechert, \& Meyer, 2005; Kwapil, Miller, Zinser, Chapman, \& Eckblad, 2000). The HPS has demonstrated both good reliability (r=0.81, Eckblad, \& Chapman, 1986; Klein, Lewinsohn, \& Seeley, 1996) and internal consistency $(\alpha=0.89$, Meyer, Druke, \& Hautzinger, 2000). This measure was included to allow investigation of whether the impact of experimental manipulation was greater amongst those with higher levels of hypomanic personality.

Godin Leisure-Time Exercise Questionnaire (GLTEQ, Godin, \& Shephard, 1985; 1997). A subjective measure of the amount of leisure time exercise lasting at least 15 minutes that an individual has participated in over the last seven days, included to characterise the sample in terms of their recent activity levels. A total exercise index is calculated by weighting each frequency by intensity. The reliability of the GLTEQ is reported to be good over two weeks ( $r=0.74-0.81$, Godin, \& Shephard, 1985; Salis, Buono, Roby, Micale, \& Nelson, 1993) and one month ( $\mathrm{r}=0.62$, Jacobs, Ainsworth, Hartman, \& Leon, 1993). It has demonstrated moderate validity with an accelerometer $(\mathrm{r}=0.32)$ and the percentage of body fat $(\mathrm{r}=-0.43)$ and a strong correlation with maximum ventilation $(\mathrm{r}=0.56)$.

Self-Reported Physical Activity Index (SRPA, Jurca et al., 2005; Mailey et al., 2010; McAuley et al., 2011). A measure used along with gender, age, Body Mass Index (BMI) and resting HR within a validated formula to calculate cardiovascular fitness (CRF) levels for the sample. Participants are asked to choose one of five activity 
categories that best describe the usual pattern of daily physical activity. CRF levels estimated from the equation using the SRPA have been found to be strongly positively correlated with two other measures of CRF. The "gold standard" maximal graded exercise test $(\mathrm{GXT})(\mathrm{r}=0.66)$ and the sub-maximal Rockport 1-mile walk test $(\mathrm{r}=0.67$, Mailey et al., 2010; McAuley et al., 2011).

Blood pressure monitor. A blood pressure monitor (AND Digital Blood Pressure Monitor) was used at the start of the testing.

Height and weight. An electronic column scale (SECA 704) and a height rod (SECA 213) were both used to measure the height and weight of participants. These variables were used to calculate BMI values for the sample.

Dependent Variables. Of these, self-reported approach motivation was the primary dependent variable. In line with secondary hypotheses we also measured a set of self-reported mania-related symptoms, plus a behavioural measure (motor activity). Finally we measured mood valence and arousal to allow comparison of our findings to previous studies of the effects of PA upon mood.

Behavioural Engagement Scale (BES, Krauss, Depue, Arbisi, \& Spoont 1992). A five item self-report measure of state behavioural engagement/AM levels. Participants are required to rate current feelings for each of the 5 items in turn (A. feelings of energy, B. optimism, C. mood, D. thought liveliness, and E. excitement) using a 10 point scale. Scores can potentially range between 0 and 50 with lower scores indicating higher levels of AM. The BES has demonstrated high internal consistency across 28 time points, $(\alpha=0.85-0.94$, Wright, Lam \& Brown, 2008). 
Manic Symptoms Questionnaire (MSQ). Using a nine point scale, participants completed a questionnaire on a number of mania related symptoms ${ }^{2}$ at five points during the testing. This procedure has been used previously by Pronin and Wegner (2006). The MSQ also included an item on speed of thoughts that was completed at the same five time points.

Motor activity. At the same time points as completing the MSQ, participants' motor activity was measured via an accelerometer (GENEActiv) worn on the wrist of the hand not used for writing in order to look at a potential objective measure of increased approach motivation. An accelerometer is a device which detects and logs movement intensity and duration. Prior to the testing, the accelerometer was synced to the local time of a computer in the testing room and set to collect triaxial data at a sampling frequency of $100 \mathrm{~Hz}$.

During the testing, the time was noted by the experimenter at the outset of completing each of the five MSQs and this time was later used during the analyses to identify the correct accelerometer data. The mean motor movement score of a 60 second period (6000 data points) for each of the axes ( $\mathrm{x}, \mathrm{y}$ and $\mathrm{z}$ ) was calculated and then the overall mean of these three was used for further analyses detailed in the results.

Self-Assessment Manikin (SAM; Lang, 1980). A nine point self-rating scale that consists of a graphic figure representing three dimensions involved in emotion: valence, arousal, and dominance. Only the valence and arousal subscales were used to measure changes in these domains during the testing period. Correlations of $r=.937$ for valence and $r=.938$ for arousal have been reported for a set of pictures using the Semantic Differential Scale (Mehrabian, \& Russell, 1974), and the SAM (Bradley, \& Lang, 1994).

\footnotetext{
${ }^{2}$ MSQ includes current levels of alertness, jitteriness, tiredness, attentiveness, activeness, strength, powerfulness, determination, creativity, insightfulness and inspiration.
} 


\section{Laboratory Approach Motivation Induction Procedure}

Participants participated in a laboratory AM induction adapted from a widely used procedure developed by Velten (1968) and used more recently by Pronin and Wegner (2006). Standardised instructions were provided and then a series of 58 statements that became increasingly elating in content were presented using Microsoft PowerPoint. Each statement scrolled into view one letter at a time from the bottom of the screen stopping in the middle of a yellow background and was presented in large black print (Arial Rounded 48-point font). Once participants had initiated the computer task, the statements advanced at a speed of 40 ms per letter with an additional $320 \mathrm{~ms}$ between slides. This procedure has been used to successfully induce feelings of high energy, power, creativity, grandiosity and perceived increased thinking speed (Pronin, \& Wegner, 2006).

\section{Experimental Manipulation}

Participants were randomly allocated to one of three conditions of differing intensities (control, moderate exercise and vigorous exercise). An author with no direct involvement in data collection created the randomisation sequence using a random number generator, then produced a series of sealed envelopes containing each participant's allocation. Intensity was coded using the classification system of the American College of Sports Medicine (ACSM). In this system, intensity can be classified in a number of ways including using heart rate, or perceived exertion (Howley, 2001).For the control condition participants were asked to read a magazine of neutral content for 15 minutes whilst sitting on a cycle ergometer. Participants in the moderate and vigorous exercise conditions completed a 15 minute period of exercise on a cycle ergometer at an experimenter desired level of intensity. The moderate condition had a target RPE of 11 and target HR of $65-75 \% \mathrm{HRmax}^{3}$ and the vigorous condition had a target

\footnotetext{
${ }^{3}$ HRmax calculated at 220-HR (Hills, Byrne \& Ramage, 1998)
} 
RPE of 15 and target HR of 76-90\% HRmax. Participants were asked to maintain a consistent level of perceived exertion throughout the activity phase of the experiment. The same cycle ergometer (Monark Ergonedic 874E) was used for the all three conditions.

\section{Manipulation Checks.}

Borg Rating of Perceived Exertion Scale (RPE, Borg, 1998). A 15 point scale used to quantify the self-rated perceived exertion levels of participants. Judgements are required to be based on an amalgamation of all physical sensations such as increased heart rate, respiration, sweating, and muscle fatigue. The RPE scale has been found to be a fairly good estimate of actual heart rate with strong correlations between RPE ratings and the indices of intensity and HR ( $\mathrm{r}=0.62)$, percentage of maximal aerobic capacity $(r=0.64)$, oxygen consumption $(\mathrm{r}=0.63)$, ventilation $(\mathrm{r}=0.61)$ and respiratory rate $(0.72$; Chen, Fan, \& Moe, 2002). Regular reports of RPE by the participant were used to ensure that participants were exercising at the intended intensity.

Heart rate (HR) monitor. Each participant wore a chest monitor (Polar Electro T31) throughout the testing which transmitted heart rates to a separate wrist unit held by the experimenter (Polar FT1). Resting HR was logged whilst the participants completed the initial questionnaires by calculating the mean of five heart rates recorded over a two and a

half minute period. HR was then recorded every three minutes during the activity (at the same time as gaining RPE ratings) for a total of five times. HR was included in order to retrospectively establish whether the conditions differed in intensity as intended.

\section{Procedure}

Prior to starting recruitment, ethical approval was sought and granted from the Research Ethics Committee at the School of Psychology, University of Exeter. All 
participants gave informed consent to take part and were required to complete an online version of the HPS prior to testing.

On attending the testing session participants were fitted with an accelerometer and heart rate band and completed a short, non-standardised self-report form to obtain gender, age, ethnicity, marital status, educational level and exercise preference, as well as standard risk assessment forms for the testing laboratory, and symptom measures (HADS and ASRM). Finally, the blood pressure, weight and height of participants were measured to calculate BMI scores. Participants meeting the inclusion criteria based on the presence of physical health comorbidities and baseline HADS and ASRM score then continued in the study and completed the GLTEQ, SRPA, MSQ, BES and SAM, and whilst doing this, a HR measure was taken at five 30 second intervals in order to calculate an accurate resting HR. Those who did not meet the inclusion criteria were offered a full debrief. Participants then completed the AM induction. Following this, a second BES and SAM rating was taken, and a second MSQ was completed. The experimenter then opened the appropriate randomisation envelope and proceeded to follow the protocol associated with the allocated experimental condition.

In all conditions except the control condition, participants were asked to warm up by pedalling for two minutes on a cycle ergometer at $30 \%$ of age-predicted HR max (calculated from resting HR). Following this warm-up period, the conditions began. Following the start of each condition, participants provided exertion levels at minutes $3,6,9,12$, and 15 post onset of experimental condition using a poster-size version of Borg's RPE scale which was situated in front of the cycle ergometer. HR was also taken at these time points. Participants provided a third and fourth BES and SAM rating at minutes 5 and 10 after the start of the condition. 
Immediately following the conclusion of the condition, participants provided a fifth BES and SAM score along with rating levels of wanting to continue with the activity ( 0 being not at all and 10 being very much). After a 2-minute cool down cycle at $30 \%$ of age-predicted HR max (or 2 minutes further magazine reading in the sedentary group) participants were asked to complete a third MSQ. Finally, participants completed a sixth BES and SAM and a fourth MSQ at a 5 minute follow up and a seventh BES and SAM and a fifth MSQ at a 10min follow-up from the end of the condition.

\section{Statistical Analysis}

Data was analysed using IBM SPSS Statistics (Version 19) for Windows Vista. Prior to the analysis, all data were visually inspected using histograms. Transformation of data was not considered necessary because of the relatively normal distributions. . In multivariate analyses, data found to violate Levene's test of homogeneity of variances were transformed using the LG10 function and if this did not improve then a non-parametric test was used. When no non-parametric equivalent analyses was available, such as with a mixed design ANCOVA, data which could not be transformed were still included in these tests as it has been argued that these tests are robust enough to account for deviations from normality (Field, 2009).

\section{Results}

In total 72 participants were recruited. Seven did not attend the testing stage of the study and four did not respond to the induction procedure described later. Hence, 61 participants were tested, ranging in age from 18 to $40(M=20.05, S D=3.67)$. Thirty eight were female. In terms of ethnicity 49 participants were White British, five were of other white ethnic origin, one participant was dual White/ Asian origin, one participant was Black British, one participant Black African, and four participants were Chinese. As expected, a large majority of the participants' highest level of education was currently attending 
university or equivalent $(n=55)$ with two having completed university and four having completed a postgraduate qualification. Means and standard deviations for scores on all dependent variables are provided in Table 1.

\section{TABLE 1}

Table 1 displays means and standard deviations for baseline variables. One-way ANOVAs were run comparing the three conditions on these. The conditions were found to differ on the baseline characteristic of fitness score, $F(2,58)=3.82, \mathrm{p}=.028$. The conditions were also found to differ on baseline levels of three dependent variables: AM levels (BES scores) $F(2,58)=3.33, \mathrm{p}=.045$, thought speed $F(2,58)=3.29, \mathrm{p}=.044$, and SAMS valence scores $F(2,58)=10.46, \mathrm{p}<.001$. A Pearson's chi square analyses was used for gender data due to its categorical nature and revealed that gender was not evenly distributed across the three conditions, $X^{2}(2, N=61)=7.07, p=0.028$. These variables were therefore used as covariates during subsequent analyses. Means and standard deviations for all baseline data are provided in Table 2.

\section{TABLE 2}

\section{Impact of AM induction procedure}

In order to establish the success of the laboratory AM induction procedure, repeated measures t-tests were conducted comparing the scores on the dependent variables before and after the induction task. It was found that following the task, participants reported increased scores for mania symptoms, $t(60)=7.42, p<.001$, thought speed, $t(60)=6.22, p<.001$, AM levels, $t(60)=13.06, p<.001$, valence, $t(60)=5.87, p<.001$, and arousal, $t(60)=4.14, p<$ .001 , suggesting that the induction was successful The results of participants with AM levels the same or lower following the laboratory induction were excluded from the study $(n=4)$. This is because the study was interested in the impact of exercise on participants already experiencing an increase in AM levels. 
One-way ANOVAs were conducted to see if there were significant differences between the three conditions in terms of the impact of the AM induction on a number of variables. Randomisation had not occurred at this point it was expected that the impact would not differ across conditions. The independent variables were the three conditions and the dependent variables were the post induction scores for AM, valence, arousal, mania symptoms, thought speed and psychomotor activity levels . There was a significant effect of condition on changes in mania symptoms, $F(2,58)=4.29, \mathrm{p}=.018$, thought speed, $F(2,58)$ $=4.15, \mathrm{p}=.021$, BES, $F(2,58)=9.31, \mathrm{p}<.001$, valence, $F(2,58)=3.42, \mathrm{p}=.039$, and arousal $F(2,58)=3.56, \mathrm{p}=.035$. This reported difference is likely to be due to chance as random assignment to condition had not occurred by this point in the procedure. Therefore, these change scores were used as covariates within the subsequent main analyses (e.g., valence change score used as a covariate within main analyses of the impact of experimental condition upon valence).

\section{Manipulation Check}

In order to establish the validity of the three conditions in terms of intensity, one-way ANOVAs were conducted to establish that there were significant differences between the three conditions. The independent variable was the three conditions and the dependent variables were the average HR and RPE ratings over the course of the activity. The results indicated that there was a significant effect of condition on the RPE levels, $F(2,58)=699.72$, $\mathrm{p}<.001$, and HR, $F(2,58)=117.13, \mathrm{p}<.001$.

\section{Main Analyses}

In all mixed design ANOVA models the following were included as covariates due to being significantly different across conditions at baseline as reported above: gender, fitness levels, AM levels, valence score, thought speed (excluding one of these three if it replicated the dependent variable), pre/post induction score change in the dependent variable and 
hypomanic traits as measured by the total HPS score. Where post-hoc comparisons were conducted a Bonferroni-corrected alpha level of $\mathrm{p}<.017$ was adopted.

\section{Hypothesis 1.}

AM Levels. A mixed design ANCOVA was used to establish if the exercise and sedentary conditions differed in terms of AM levels following the exercise manipulation. The dependent variable was AM level (with an increase indicated by a decreased BES score) over six time points (at the start of activity, five minutes into activity, 10 minutes into activity, end of activity, 5 minute follow-up and 10 minute follow-up) and the independent variable was the condition (sedentary, moderate or vigorous exercise). Significant main effects of Time, $F(2.49,129.8)=4.92, \mathrm{p}=.005$, and Condition, $F(2,52)=7.28, \mathrm{p}=.002$, were found. These should be interpreted in the light of a significant Time $\mathrm{x}$ Condition interaction, $F(4.99,129.8)$ $=3.24, \mathrm{p}=.009$. Figure 1 illustrates the pattern of results for AM levels across time, by conditions. In partial support of the hypothesis, AM levels in the vigorous condition appear to have increased over time in comparison to the control and moderate conditions. Confirming this, polynomial contrasts of the Time $\mathrm{x}$ Condition interaction revealed a significant linear trend, $F(2,52)=6.62, p=.003$. A test of simple contrasts comparing AM levels at the start of activity to all subsequent time points revealed that five minutes into and at the end of the activity, the three conditions did not differ in terms of change in AM levels, but ten minutes into, $F(2,52)=3.32, p=.044$ and at the five, $F(2,52)=6.58, p=.003$ and ten minute recovery they did, $F(2,52)=7.19, p=.002$.

\section{FIGURE 1}

To deconstruct the effect further the ANCOVA was repeated, this time including the vigorous and control conditions only. Consistent with hypothesis one there was a significant main effect of Condition, $F(1,33)=10.52, p=.003$, in the context of a significant Time $\mathrm{x}$ Condition interaction, $F(2.45,80.72)=5.23, p=.004$. A test of simple contrasts comparing 
AM levels at the start of activity to all subsequent time points revealed that five minutes into the activity, the two conditions did not differ in terms of change in AM levels, but did ten minutes in, at the end of the activity and at five and ten minute recovery such that participants in the vigorous condition reported greater increase in AM levels from baseline than did those in the control condition.

Contrary to hypotheses one, a similar planned comparison of the moderate and control conditions revealed a significant main effect of Time, $F(2.62,86.51)=5.06, p=.004$, but no significant effect of Condition, nor a significant Time x Condition interaction. Finally, a post-hoc mixed model ANCOVA including only the vigorous and moderate conditions revealed a main effect of condition such that AM levels were significantly higher in the vigorous relative to the moderate condition, $F(1,32)=7.46, p=.010$. There was also a significant main effect of Time, $F(2.10,67.24)=5.16, p=.007$, but no significant Time $\mathrm{x}$ Condition interaction.

Valence. A mixed design ANCOVA was conducted on reported valence levels. The dependent variable was Valence scores over six time points and the independent variable was condition. There was a significant main effect of Time, $F(3.46,180.07)=$ $8.96, \mathrm{p}<.001$, and Condition, $F(2,52)=6.58, \mathrm{p}=.003$, in the context of a significant Time $\mathrm{x}$ Condition interaction, $F(6.93,180.07)=3.33, \mathrm{p}=.002$. Polynomial contrasts of the Time $\mathrm{x}$ Condition interaction revealed significant linear, $F(2,52)=6.58, \mathrm{p}=.007$, and quadratic, $F(2,52)=4.45, \mathrm{p}=.016$, trends. A test of simple contrasts comparing valence scores at the start of activity to all other time points revealed that five minutes into and at the end of the activity, the three conditions did not differ significantly in terms of change in valence scores but did at ten minutes in, $F(2,52)=3.45, \mathrm{p}=.039$ and at five, $F(2,52)=4.86, \mathrm{p}=.012$ and ten minute recovery $F(2,52)=4.47, \mathrm{p}=.016$. FIGURE 2 
To deconstruct the effect, the above ANCOVA was repeated, including the vigorous and control conditions only. This revealed a significant main effect of Condition, $F(1,33)=$ $6.22, p=.018$, and Time, $F(3.02,99.6)=12.44, p<.001$, in the context of a significant Time x Condition interaction, $F(3.02,99.6)=3.59, p=.016$. As hypothesised, overall scores were higher in the vigorous condition. A test of simple contrasts comparing valence scores at the start of activity to all other time points revealed that the two conditions differed in change in valence scores at the five, $F(1,33)=13.02, p=.001$ and ten minute recovery points, $F(1,33)$ $=12.51, p=.001$ such that those in the vigorous condition demonstrated a greater increase in positive mood from baseline than did those in the control condition.

A planned comparison of the moderate and control conditions revealed a significant Time $\mathrm{x}$ Condition interaction, $F(3.80,125.46)=4.94, p=.001$. As hypothesised, a test of simple contrasts comparing valence scores at the start of activity to all other time points revealed that ten minutes in to the activity those in the moderate condition reported greater increase in positive mood from baseline, $F(1,33)=4.75, p=.037$, but not at any time period after this.

Finally, a post-hoc ANCOVA including only the vigorous and moderate conditions revealed a significant main effects of Condition, $F(1,32)=9.44, p=.004$ and Time, $F(2.73$, $87.32)=6.08, p=.001$, but no significant Time $\mathrm{x}$ Condition interaction. Thus valence levels were found to be higher overall during exercise for those in the vigorous condition as compared to those in the moderate condition.

Arousal. A mixed design ANCOVA was conducted on reported arousal levels. There was a significant main effect of Time, $F(3.06,155.82)=10.03, \mathrm{p}<.001$, and Condition, $F(2,51)=12.71, \mathrm{p}<.001$, in the context of a significant Time $\mathrm{x}$ Condition interaction, $F(6.11,155.82)=8.84, \mathrm{p}<.001$. Polynomial contrasts revealed a significant quadratic trend for the interaction, $F(2,51)=19.11, \mathrm{p}<.001$. As 


\section{EXERCISE AND APPROACH MOTIVATION}

hypothesised a test of simple contrasts comparing arousal scores at the start of activity to all subsequent time points revealed that the three conditions differed significantly in terms of change in arousal levels at five, $F(2,51)=4.09, \mathrm{p}=.023$, and ten minutes into the activity, $F(2,51)=8.92, \mathrm{p}<.001$, at the end of the activity, $F(2,51)=15.98, \mathrm{p}$ $<.001$ and at the five minute recovery period, $F(2,51)=4.47, \mathrm{p}=.016$ but not at the ten minute recovery.

\section{FIGURE 3}

A planned comparison of only the vigorous and control conditions, repeating the above ANCOVA, revealed a significant main effect of Condition, $F(1,32)=27.76, p<.001$, and of Time, $F(2.76,88.42)=8.13, p<.001$ in the context of a significant Time $x$ Condition interaction, $F(2.76,88.42)=11.81, p<.001$. As hypothesised, a test of simple contrasts comparing arousal scores at the start of activity to those for subsequent time points revealed that the two conditions differed significantly in terms of change in arousal levels at five $F(1$, $32)=5.24, p=.029$, ten minutes into the activity, $F(1,32)=12.41, p=.001$ and at the end of the activity, $F(1,32)=25.28, p<.001$ but not at five and ten min recovery, such that arousal scores increased from baseline in the vigorous condition, relative to the control condition.

A planned comparison of the moderate and control conditions did not reveal a significant main effect of Condition but a significant effect of Time, $F(2.81,89.75)=3.20, p$ $=.030$, was found in the context of a significant Time $\mathrm{x}$ Condition interaction, $F(2.81,89.75)$ $=3.70, p=.017$, such that arousal levels increased during activity in the moderate condition relative to the control condition, then decreased post activity, resulting in significantly greater decrease in arousal scores from baseline in the moderate condition by the final time point, $F(1,32)=6.92, p=.013$ 
Finally, a post-hoc ANCOVA including only the vigorous and moderate revealed a significant effect of Time, $F(3.39,105.05)=9.68, p<.001$, such that arousal scores decreased over time but no main effect of condition or Time $\mathrm{x}$ Condition interaction.

Thought Speed. A mixed design ANCOVA was conducted on perceived thought speed following the activity. The dependent variable was perceived thought speed scores over three time points (post activity, 5 minutes into recovery and 10 minutes into recovery), with condition as the independent variable. The main effects of Time and Condition, were not found to be significant and contrary to the hypothesis, the Time $\mathrm{x}$ Condition interaction was not significant indicating that reported thought speed was not significantly different for the three conditions over different time points.

Mania Symptoms. A mixed design ANCOVA was conducted on reported mania symptoms following the activity. The dependent variable was total score on the MSQ over three time points as above, with condition as the independent variable. The main effect of Time was not found to be significant, but a significant main effect was found for Condition, $F(2,51)=3.58, \mathrm{p}=.035$. No significant Time $\mathrm{x}$ Condition interaction was found. Contrary to our hypothesis the vigorous and moderate conditions did not differ significantly from the control condition.

A post-hoc comparison of the vigorous and moderate conditions revealed a significant main effect of Condition, $F(1,31)=9.29, p=.005$, with higher levels of mania symptoms found in the vigorous condition.

Motor Activity. A mixed design ANCOVA was conducted on motor activity following the activity. The dependent variable was mean motor movement score (across all three axes) at each of the three time periods and the independent variable was condition. No significant effects were found. 
Hypothesis 2. Mixed design ANCOVAs were conducted on the data to investigate whether hypomanic traits measures by the HPS moderated the effects reported in Hypotheses 1. The same variables entered into the models used for hypotheses 1 were used in the analyses reported here, however an additional three-way interaction between HPS scores, Condition (three levels) and Time (6 or 3 levels) was included.

The analyses did not reveal a significant HPS x Condition x Time interaction for AM levels, Valence scores Arousal scores, mania symptoms or psychomotor activity. A significant HPS x Condition x Time interaction was found for thought speed, $F(2.64,66.09)$ $=3.34, p=.029$. Simple contrasts on this interaction revealed that participants' thoughts speed was significantly different between post activity (level 1) and the five minute recovery point (level 2), $F(2,50)=5.05, p=.010$, such that it increased for vigorous condition and reduced in the control and moderate conditions.

The sample was separated into two subsamples, one containing those scoring below the median HPS score ("low HPS"), and the other containing those scoring above ("high HPS"). Repeating the ANCOVA on thought speed for both subsamples separately revealed a significant Condition $\mathrm{x}$ Time interaction for low HPS scorers $F(2.59,29.73)=4.91, p=.009$, but not for high HPS scorers $F(2.70,21.59)=2.36, p=.105$.

Hypothesis 3. A Kendall's tau non parametric correlation did not reveal a significant correlation $r=0.12, p=0.453$, between the time taken for AM levels to return to baseline and HPS scores.

Hypothesis 4. A hierarchical regression was conducted on the relationship between hypomanic symptoms and wanting to continue with the activity for the two exercise conditions. The predictors were the total HPS scores and condition $(0$ for moderate and 1 for vigorous) and the dependent variable was participants' ratings of wanting to continue with the activity. Condition and HPS were entered in the model 
first followed by the Condition x HPS interaction in the second step. Analyses did not reveal a significant correlation between wanting to continue with the activity and HPS scores, or between wanting to continue with the activity and a Condition x HPS interaction.

\section{Discussion}

The main aim of the current study was to test the hypothesis that - in an unselected student sample - exercise would have an impact on the maintenance of AM levels once elevated. We found that, following induction of heightened AM, those participants who engaged in vigorous exercise appeared to experience prolonged elevation of approach motivation relative to those in the sedentary condition. This is in keeping with conceptualisations of the approach system, whereby it is activated by striving towards potential reward or an approach goal, a feature that is often present within exercise that requires participants to work hard towards a particular target.

To our knowledge this is the first study to explore the effects of exercise upon the general population distinct from positive mood or arousal levels. Our findings regarding the effects of exercise on arousal and valence serve as a link between our study and the existing literature. In keeping with an effect observed in previous research (Ekkekakis \& Petruzzello, 1999; Hall et al., 2002), following termination of exercise we observed a sharp increase in affective valence, relative to the sedentary condition. Also in keeping with previous findings (Hall et al., 2002) those in the exercise conditions reported increased arousal during exercise, followed by a reduction.

In addition to exploring effects upon AM, valence and arousal, we investigated whether engaging in exercise when in a high mood state affects other variables linked to mania symptomatology, namely motor activity, thought speed and felt internal states typical of mania (as measured by the MSQ). In contrast to the findings for AM, valence and arousal 
we did not find that changes in these variables over time differed between the three groups. However, we did find those in the vigorous condition to show elevated levels of thought speed and mania-like symptoms throughout exercise, as compared to those in the moderate condition.

Overall, our findings suggest that exercising whilst in a state of heightened approach motivation can promote both sustained AM and positive, activated mood. Although we cannot necessarily consider the high AM levels induced in this study to be analogous to mania, our findings are consistent with our proposed upward spiral model described earlier. In line with this, it would be expected that individuals who present with a higher number of hypomanic traits would show greater disturbance within the BAS impacting on levels of AM, and other associated factors. However, contrary to our prediction, results indicated that hypomanic traits did not moderate the presence of AM levels, valence, arousal, mania symptoms or psychomotor activity. This finding is surprising because it would be expected that hypomanic traits would lead to greater dysregulation of an individual's BAS and hence moderate activities that perturb the BAS such as exercise. Previous research has shown that hypomanic traits are positively related to BAS scores (Carver, \& Johnson, 2009; Meyer et al., 1999) and are predictive of manic episodes, substance use, and involvement in pleasurable but potentially harmful leisure activities (Krumm-Merabet, \& Meyer, 2005; Kwapil, Miller, Zinser, Chapman, Chapman, \& Eckblad, 2000; Meyer, \& Hautzinger, 2003; Meyer, \& Hoffman, 2005) as well as the course and severity of manic symptoms over time (Meyer et al., 2001). Additionally, the BAS subscale of the BIS/BAS Scale (Carver, \& White,1994) has been reported to be positively correlated with the HPS scale (Jones, \& Day, 2008; Meyer, \& Hofmann, 2005). Similarly, research suggests that mania is highly correlated with psychomotor activity (Johnson, Edge, Holmes, \& Carver, 2012), such that physical activity is 
considered by some to be a more reliable criterion than changes in mood for making a diagnosis (Akiskal, \& Benazzi, 2005).

Hypomanic traits were found to moderate reported thought speed following an activity in different conditions however, this effect seemed to be driven by an increase in thought speed for low HPS scorers after vigorous exercise compared to the other conditions. A moderating effect of HPS scores was not found for participants with higher hypomanic traits.

According to the BAS model, exercise can act as a trigger of the approach system; once approach motivation is elevated, the individual is more likely to be attracted to and engage in further exercise (if it involves an element of goal-pursuit), thus promoting further AM escalation. This process is hypothesised to be particularly marked in individuals with Bipolar Disorder, in whom homeostatic mechanisms for regulating approach motivation may be less strong (Depue \& Iacono, 1989; Depue et al., 1987), hence findings of increased attraction to exercise in individuals during hypomania (Wright et al., 2011), and an association between exercise addiction and hypomanic personality traits (Meyer, Rahman, \& Shepherd, 2007). Our findings were not consistent with this hypothesis: we did not find the predicted association between HPS score and desire to continue exercising. It is possible that our method of measuring individuals' desire to continue with the activity may not have addressed the construct of interest: we used an unvalidated, single-item measure of desire to continue exercising. Scores on the HPS have been found to be positively correlated with BAS scores (Jones, \& Day, 2008; Meyer, \& Hofmann, 2005) and are predictive of manic episodes, substance use, and involvement in pleasurable but potentially harmful leisure activities (Krumm-Merabet, \& Meyer, 2005; Kwapil, Miller, Zinser, Chapman, Chapman, \& Eckblad, 2000; Meyer, \& Hautzinger, 2003; Meyer, \& Hoffman, 2005) as well as the course and severity of manic symptoms over time (Meyer et al., 2001). Nevertheless it is possible that 
levels of hypomanic traits, and crucially, vulnerability to Bipolar Disorder, amongst participants were not high enough to observe the predicted effects. Replication of the study with a sample of individuals with and without Bipolar Disorder would provide a better test of the moderating role of Bipolar Disorder vulnerability upon the impact of exercise upon AM. Furthermore, the induction of AM in our study may have been insufficient in magnitude to result in differential response in more vulnerable individuals: although only small number of individuals did not experience heightened AM levels just under a quarter only experienced a change of one point. A more convincing test would involve asking vulnerable individuals to engage in exercise (versus comparator conditions) whilst in a naturally-occurring mild hypomanic state, however given the pre-existing concerns about the potential of vigorous exercise to contribute to hypomania, this methodology raises significant ethical issues. Such an approach might be best suited to exploring the impact of potentially beneficial (rather than deleterious) forms of physical activity upon hypomania, potentially with a patient group receiving high-intensity clinical care, for example within an inpatient unit. Importantly though, even if this effect is not found to be more pronounced in individuals with BD, the finding that vigorous exercise sustains high AM levels - if replicated in this population - has considerable significance the management of hypomania: when levels of high mood and approach motivation are greatly disturbed it most likely becomes more important than usual to avoid substances or activities that sustain or increase the state.

More generally the reliability and generalizability of our findings is subject to a number of limitations.

First, previous research exploring the effect of exercise on positive activated affect has employed activity of longer duration: Reed and Ones (2006) have reported that a duration of 30 to 35 minutes produced the largest effect sizes within an extensive meta-analysis. This raises the question of whether the findings of our study would have been replicated had a 
different duration of exercise been employed. Indeed, in delineating helpful versus unhelpful exercise behaviour during hypomania, the impact of exercise duration may need to be investigated particularly with regard to the durations that individuals would naturally engage in.

Second, the positioning of the accelerometer when measuring motor activity may have limited our ability to observe experimental effects. Rather than using a device that is attached to the wrist, greater sensitivity may have been achieved by using one positioned upon the torso. However, given that participants were to be seated for the critical recording periods the decision made to focus upon arm movement in particular. Relatedly, although we measured levels of physical fitness as baseline, we did not measure certain variables that may have affected levels of motor activity, such as stimulant ingestion.

Finally, randomisation was not entirely successful: the groups were found to differ significantly on some variables at baseline, despite the use of a robust randomisation procedure. Although this imbalance was taken into account statistically, a methodological solution would be preferable in future (such as stratification on key variables).

To inform understanding of the relationship between approach motivation and exercise in the general population it is necessary to replicate these results with a larger and more diverse sample in terms of age, socio-economic status and education. Inclusion of a control sample, who had not experienced the laboratory induced hypomanic state would also be beneficial in determining the impact that physical activity would have on AM even in the absence of an induction. Consideration of the limitations raised above would also be recommended, particularly in terms of extending the duration of exercise and recovery periods. Following on from this, the effect of different intensities of exercise in a sample with diagnosed BD could be investigated although this presents ethical challenges due to the possible harmful effects of vigorous exercise. Current literature suggests that individuals with 
BD (Harmon-Jones et al., 2008; Johnson et al., 2012) and student populations at high risk for mania (Harmon-Jones et al. 2002) are more likely to expend effort, work harder and sustain effort for longer when offered a reward and this may another future area of interest. It would be important to look at the impact of different intensities of exercise on mania as well as hypomania because it may be that individuals with a more dysregulated BAS may react differently to exercise and as such may experience an even more beneficial or detrimental outcome.

In line with the reported comments raised by participants in the study reported by Wright (2011), it would be important to consider the impact of different precipitating mood states at the outset of the exercise as well as the impact of different contexts (outside versus inside) on the maintenance of AM levels. Increase in AM through physical activity is not necessarily deleterious: during low moods in particular this may be desirable and may reflect increase in positive mood and energy levels.

Thus in addition to identifying the profile of exercise which may, in some individuals, lead to untoward effects, a valuable future goal of this line of research should be to determine patterns of physical activity that have the potential to down-regulate hypomanic states, relive depressive states, and enhance mood stability.

In conclusion our findings support the hypothesis that exercise can lead to continued elevations in approach motivation and positive, activated mood when carried out during a period of activated, elevated mood, but that this pattern appears to be relatively specific to vigorous exercise. In addition mania-like symptoms were found to be elevated during vigorous exercise relative to moderate exercise. Tests of these effects within individuals with Bipolar Disorder, and within ecologically valid hypomanic states, are required before clinical practice or exercise guidance can be reliably informed. However, our findings join an emerging body of literature which suggests that the impact of physical activity upon 
hypomania may vary depending upon features of the activity. Considering this literature as a whole, we recommend that individuals with Bipolar Disorder are encouraged by their clinicians to observe the particular relationship between exercise and their own symptoms in order to make informed choices about engaging in PA that maximises both physical and mental health benefits, whilst minimising deleterious effects. 


\section{References}

Akiskal, H. S., \& Benazzi, F. (2005).Optimizing the detection of bipolar II disorder in outpatient private practice: Toward a systematization of clinical diagnostic wisdom. Journal of Clinical Psychiatry, 66, 914-921.

Alloy, L. B. \& Abramson, L. Y. (2010). The role of the behavioral approach system (BAS) in bipolar spectrum disorders. Current Directions in Psychological Science, 19(3), 189194.

Altman, E. G., Hedeker, D., Peterson, J. L., \& Davis, J. M., (1997). The Altman Self-Rating Mania Scale. Biological Psychiatry, 42(10), 948-955.

American Psychiatric Association. (2013). Diagnostic and statistical manual of mental disorders (5th ed.). Arlington, VA: American Psychiatric Publishing.

Benazzi, F., \& Akiskal, H. S. (2003). The dual factor structure of self-rated MDQ hypomania: Energized-activity versus irritable-thought racing. Journal of Affective Disorders, 73, 59-64.

Borg, G. (2008). Perceived exertion and pain scale. Champaign, IL: Human Kinetics.

Brisswalter, J., Collardeau, M., \& Rene, A. (2002). Effects of acute physical exercise characteristics on cognitive performance. Sports Medicine, 32, 555-566.

Carver, C. S., \& Johnson, S. L. (2009). Tendencies toward mania and tendencies toward depression have distinct motivational, affective, and cognitive correlates. Cognitive Therapy and Research, 33, 552-569.

Carver, C. S., \& White, T. L. (1994). Behavioral inhibition, behavioral activation, and affective responses to impending reward and punishment: The BIS/BAS scales. Journal of Personality and Social Psychology, 67, 319-333.

Depue, R. A. \& Iacono, W. G. (1989). Neurobehavioral aspects of affective disorders. Annual Review of Psychology, 40, 457-492.

Depue, R. A., Krauss, S. P. \& Spoont, M. R. (1987). A two-dimensional threshold model of seasonal bipolar affective disorder. In D. Magnusson, \& A. Ohman (Eds.) Psychopathology: An interactionist perspective (pp. 95-123). New York: Academic Press.

Eckblad, M., \& Chapman, L. J. (1986). Development and validation of a scale for hypomanic personality. Journal of Abnormal Psychology, 95, 214-222. 
Ekkekakis, P., Parfitt, G. \&. Petruzzello, S. J. (2011). The Pleasure and Displeasure People Feel When they Exercise at Different Intensities Decennial Update and Progress towards a Tripartite Rationale for Exercise Intensity Prescription, Sports Medicine, 41(8), 641-671.

Elliot, A. J. (1999). Approach and avoidance motivation and achievement goals. Educational Psychologist, 34(3), 169-189.

Emery, C. F., Schein, R. L., Hauck, E. R., \& MacIntyre, N. R. (1998). Psychological and cognitive outcomes of a randomized trial of exercise among patients with obstructive pulmonary disease. Health Psychology, 17, 232-240.

Ferguson, E. D. (2010). The corsini encyclopedia of psychology, (Vol. 3). New York, NY: Wiley.

Field, A. (2009). Discovering statistics using SPSS. London: Sage.

Godin, G., \& Shephard R. J. (1985). A simple method to assess exercise behavior in the community. Canadian Journal of Applied Sport Sciences. 10, 141-146.

Godin, G., \& Shephard, R. J. (1997). Godin leisure-time exercise questionnaire. Medicine and Science in Sports and Exercise, 29, 36-38.

Goodwin, K. F., \& Jamison, R. K. (2007). Manic-depressive illness: bipolar disorders and recurrent depression. Oxford University Press, New York.

Goplerud, E. \& Depue, R. A. (1985). Behavioral response to naturally-occurring stress in cyclothymes, dysthymes, and controls. Journal of Abnormal Psychology, 94, 128-139.

Gray, J. A. (1987). The psychology of fear and stress. Cambridge: Cambridge University Press.

Hall, E. E., Ekkekakis, P., \& Petruzzello, S. J. (2005). Is the relationship of RPE to psychological factors intensity dependent? Medicine and Science in Sport and Exercise, 37(8), 1365-1373.

Hansen, C. J., Stevens, L. C., \& Coast, J. R. (2001). Exercise duration and mood state: How much is enough to feel better? Health Psychology, 20, 267-275.

Harmon-Jones, E., Abramson, L.Y., Nusslock, R., Sigelman, J.D., Urosevic, S., Turonie, L.D., . . Fearn, M. (2008). Effect of bipolar disorder on left frontal cortical responses to goals differing in valence and task difficulty. Biological Psychiatry, 63, 693-698.

Harmon-Jones, E., Abramson, L. Y., Sigelman, J. D., Bohlig, A., Hogan, M. E., \& HarmonJones, C. (2002). Proneness to hypomania/mania symptoms or depression symptoms and asymmetrical frontal cortical responses to an anger-evoking event. Journal of Personality and Social Psychology, 82, 610-618. 
Hills, P., Byrne, N. M. \& Ramage, A. J. (1998). Submaximal markers of exercise intensity, Journal of Sports Sciences, 16(1), 71-76

Howley, E. T. (2001). Type of activity: Resistance, aerobic and leisure versus occupational physical activity. Medicine and Science in Sports and Exercise, 33(6), 364-S369.

Johnson, S. L., Edge, M. D., Holmes, M. K., \& Carver, C. S. (2012). The behavioral activation system and mania. Annual Review of Clinical Psychology. 8, 243-267.

Johnson, S. L., Ruggero, C. J., \& Carver, C. S. (2005). Cognitive, behavioral, and affective responses to reward: Links with hypomanic symptoms. Journal of Social and Clinical Psychology, 24, 894-906.

Jones, S., \& Day, C. (2008). Self appraisal and behavioural activation in the prediction of hypomanic personality and depressive symptoms. Personality and Individual Differences, 45, 643-648.

Judd, L. L., Akiskal, H. S., Schettler, P. J., Endicott, J., Maser, J., Solomon, D. A., .. Keller, M. B. (2002). The long-term natural history of the weekly symptomatic status of bipolar I disorder. Archives of General Psychiatry, 59(6), 530-537.

Jurca, R., Jackson A. S., LaMonte, M. J., Morrow, J. R., Blair, S. N., Wareham, N. J., ... Jakicic, J. M. (2005). Assessing cardiorespiratory fitness without performing exercise testing. American Journal of Preventative Medicine, 29(3), 185-193.

Krauss, S. S., Depue, R. A., Arbisi, P. A., \& Spoont, M. (1992). Behavioral engagement level, variability, and diurnal rhythm as a function of bright light in bipolar II seasonal affective disorder: An exploratory study. Psychiatry Research, 43(2), 147-160.

Krumm-Merabet, C., \& Meyer, T. D. (2005). Leisure activities, alcohol, and nicotine consumption in people with a hypomanic/hyperthymic temperament. Personality and Individual Differences, 38, 701-712.

Kwapil, T. R., Miller, M. B., Zinser, M. C., Chapman, L. J., Chapman, J., \& Eckblad, M. (2000). A longitudinal study of high scorers on the hypomanic personality scale. Journal of Abnormal Psychology, 109, 222-226.

Lang, P. J. (1980). Behavioral treatment and bio-behavioral assessment: Computer applications. In J. B. Sidowski, J. H. Johnson, \& T. A. Williams (Eds.) Technology in mental health care delivery systems (pp. 119-1 37). Norwood, NJ: Ablex.

Mailey, E. L., White, S. M., Wojcicki, T. R., Szabo, A. N., Kramer, A. F., \& McAuley, E. (2010). Construct validation of a non-exercise measure of cardiorespiratory fitness in older adults. BMC Public Health, 10(59), 1471-1479. 
McAuley, E., Szabo, A. N., Mailey, E. L., Erickson, K. I., Voss, M., White, S. M., Wójcicki, T. R., Gothe, N., Olson, E. A., Mullen, S. P., Kramer, A. F. (2011). Non-exercise estimated cardiorespiratory fitness: Associations with brain structure, cognition, and memory complaints in older adults. Mental Health and Physical Activity, 4(1), 5-11.

Mead, G. E., Morley, W., Campbell, P., Greig, C. A., McMurdo, M., \& Lawlor, D. A. (2008). Exercise for depression. Cochrane database of systematic reviews (Issue 4) Art. No.: CD004366.

Meyer, T. D., \& Hautzinger, M. (2003). Screening for bipolar disorders using the hypomanic personality scale. Journal of Affective Disorders, 75, 149-154.

Meyer, T. D., \& Hofmann B. U. (2005). Assessing the dysregulation of the behavioral activation system: The hypomanic personality scale and the BIS-BAS scales Journal of Personality Assessment, 85, 318-324.

Meyer, B., Johnson, S. L., \& Carver, C. S. (1999). Exploring behavioral activation and inhibition sensitivities among college students at risk for bipolar-spectrum symptomatology. Journal of Psychopathology and Behavioral Assessment, 21, 275292.

Meyer, B., Johnson, S. L., \& Winters R. (2001). Responsiveness to threat and incentive in bipolar disorder: relations of the BIS/BAS Scales with symptoms. Journal of Psychopathology and Behavioural Assessment, 23, 133-143.

Meyer, B., Rahman R., \& Shepherd, R. (2007). Hypomanic personality features and addictive tendencies. Personality and Individual Differences, 42, 801-810.

Moore, P., Wright, K., \& Taylor, A. H. (2011). The relationship of trait mood variability to the effects of acute exercise upon mood, cognition and behaviour. Unpublished manuscript, University of Exeter, Exeter.

National Institute for Health and Clinical Excellence. (2006). Bipolar disorder: The management of bipolar disorder in adults, children and adolescents, in primary and secondary care. NICE clinical guideline 38, London: National Institute for Health and Clinical Excellence.

Osei-Tutu, K. B., \& Campagna, P. D. (2005). The effects of short- vs. long-bout exercise on mood, VO-sub(2MAX), and percept body fat. Preventative Medicine, 40, 92-98.

Pronin, E., \& Wegner, D. M. (2006). Independent effects of thought speed and thought content on mood. Psychological Science, 17, 807-813.

Reed, J., \& Ones, D. S. (2006). The effect of acute aerobic exercise on positive activated affect: A meta-analysis Psychology of Sport and Exercise, 7, 477-514. 
Schneider, M., \& Graham, D. (2009). Personality, physical fitness, and affective response to exercise

among adolescents. Medicine and Science in Sport and Exercise, 41(4), 947-955.

Stanton, R., Happell, B., Hayman, M. \& Reaburn, P. (2014). Exercise interventions for the treatment of affective disorders - Research to practice, Frontiers in Psychiatry, 46(5), $1-4$.

Sylvia, L. G., Friedman, E. S., Kocsis, J. H., Bernstein, E. E., Brody, B. D., Kinrys, G., et al. (2013). Association of exercise with quality of life and mood symptoms in a comparative effectiveness study of bipolar disorder. Journal of Affective Disorders, $151,722-727$.

Thomas, S., Reading, J. \& Shephard, R. J. (1992). Revision of the physical activity readiness questionnaire (PAR-Q). Canadian Journal of Sports Science. 17(4), 338-345.

Urosevic, S., Abramson L. Y., Harmon-Jones, E., \& Alloy, L. B. (2008). Dysregulation of the behavioral approach system (BAS) in bipolar spectrum disorders: Review of theory and evidence. Clinical Psychology Review, 28, 1188-1205.

Velten, E. C., Jr. (1968). A laboratory task for induction of mood states. Behaviour Research and Therapy, 6, 473-482.

Watson, D., Wiese., D., Vaidya, J. \& Tellegen, A., (1999). The two general activation systems of affect: Structural findings, evolutionary considerations, and psychobiological evidence. Journal of Personality and Social Psychology, 76(5), 820838.

Wright, K. A., Armstrong, T., Taylor, A., \& Dean, S. (2011). 'It's a double edged sword': A qualitative analysis of the experiences of exercise amongst people with bipolar disorder. Journal of Affective Disorders, 136(3), 634-642.

Wright, K. A., Everson-Hock, E. S. \& Taylor, A. H. (2009). The effects of physical activity on physical and mental health among individuals with bipolar disorder: A systematic review. Mental Health and Physical Activity, 2, 86-94.

Wright, K. A., Lam, D. \& Brown, R. G. (2008). Dysregulation of the behavioral activation system in remitted Bipolar I Disorder. Journal of Abnormal Psychology, 117(4), 838848.

Zigmond, A. S., \& Snaith, R. P. (1983). The Hospital Anxiety and Depression Scale. Acta Psychiatrica Scandinavica, 67, 361-370. 
Zschucke, E., Gaudlitz, K., \& Ströhle. (2013). Exercise and physical activity in mental disorders: Clinical and experimental evidence. Journal of Preventative Medicine and Public Health, 46(1), 12-21. 
Mean Scores for Dependent Variables During and Post Activity

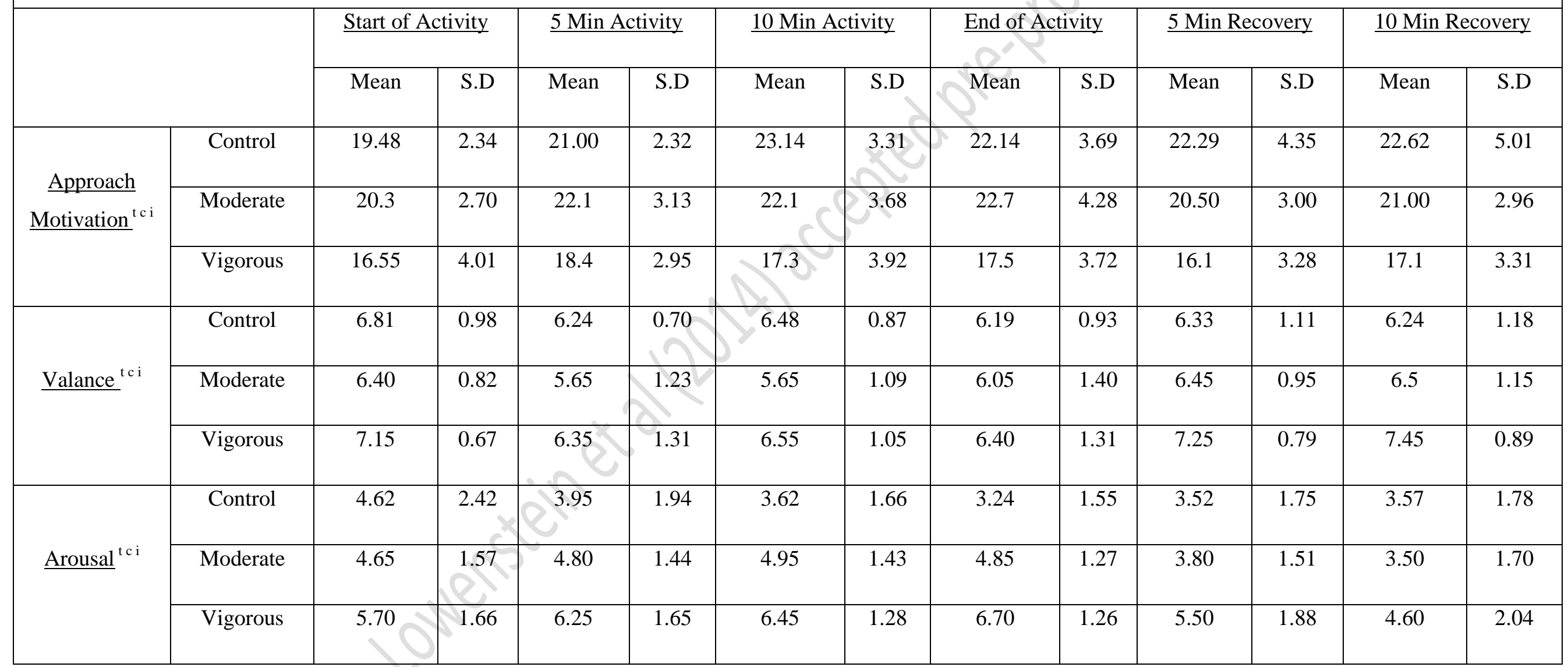


EXERCISE AND APPROACH MOTIVATION

\begin{tabular}{|c|c|c|c|c|c|c|c|c|c|c|c|c|c|}
\hline \multirow{3}{*}{$\begin{array}{l}\text { Thought } \\
\underline{\text { Speed }}^{\text {tc }}\end{array}$} & Control & 4.71 & 2.65 & - & - & - & - & 4.05 & 2.62 & 3.95 & 2.48 & 4.43 & 2.71 \\
\hline & Moderate & 5.30 & 0.73 & - & - & - & - & 5.10 & 1.02 & 4.85 & 0.93 & 4.60 & 1.14 \\
\hline & Vigorous & 6.30 & 0.87 & - & - & - & - & 5.50 & 1.64 & 5.85 & 0.99 & 5.55 & 1.05 \\
\hline \multirow{3}{*}{$\frac{\text { Current Mania }}{\text { Symptoms }^{c}}$} & Control & 59.00 & 19.57 & - & - & - & - & 51.52 & 21.75 & 50.24 & 21.97 & 52.24 & 20.32 \\
\hline & Moderate & 64.45 & 5.71 & - & - & - & - & 61.40 & 12.63 & 61.55 & 9.76 & 59.75 & 8.94 \\
\hline & Vigorous & 72.40 & 6.10 & - & - & - & & 72.00 & 13.71 & 71.6 & 13.03 & 68.95 & 13.39 \\
\hline \multirow{3}{*}{$\frac{\text { Psychomotor }}{\underline{\text { Activity }}}$} & Control & -0.30 & 0.09 & - & - & - & & -0.17 & 0.21 & -0.25 & 0.18 & -0.22 & 0.15 \\
\hline & Moderate & -0.31 & 0.20 & - & - & & - & -0.20 & 0.29 & -0.22 & 0.28 & -0.29 & 0.22 \\
\hline & Vigorous & -0.36 & 0.18 & - & - & & - & -0.37 & 0.19 & -0.4 & 0.22 & -0.39 & 0.05 \\
\hline
\end{tabular}

$\mathrm{t}$ main effect of time

c main effect of condition

${ }^{\mathrm{i}}$ interaction between time and condition 